\title{
The Forkhead Gene Family in Medaka: Expression Patterns and Gene Evolution
}

\author{
X. Shen ${ }^{\text {a }}$ J.Cui ${ }^{\text {b }}$ Y. Nagahama ${ }^{c}$ \\ ${ }^{a}$ Reproductive Genomics Group, Temasek Life Sciences Laboratory, and bSaw Swee Hock School of Public Health, \\ National University of Singapore, Singapore, Singapore; ' Laboratory of Reproductive Biology, National Institute \\ for Basic Biology, Okazaki, Japan
}

\section{Key Words}

Evolution • Expression pattern • Forkhead $\cdot$ Gene family .

Medaka $\cdot$ Phylogenetic relationship $\cdot$ Zebrafish

\begin{abstract}
Using the genome sequence of the medaka, Oryzias latipes, we examined the genomic complement of Fox genes in this organism to gain insight into the evolutionary relationships and expression patterns of this gene family. We identified 31 Fox genes by searching for Forkhead domains in the medaka genome and by polymerase chain reaction (PCR) using primers designed from protein alignments. All the medaka Fox genes are encoded in 18 subclasses as follows: 5 Fox genes in subclass $D ; 3$ Fox genes in subclass $0 ; 2$ Fox genes in each of subclass $A, B, E, F, G, I, P$, and $Q$, respectively; 1 Fox gene in each of subclass $C, H, J, K, M, N$, and $R$. The gene structures and general features are also discussed. In addition, we examined the expression patterns of some of these genes in different adult tissues and during embryonic development in medaka using quantitative PCR and in situ hybridization. The present study shows that Forkhead transcription factors play an important role during early embryonic development in medaka, and the results will enhance our knowledge in terms of Fox family evolution in different taxa.
\end{abstract}

Copyright $\odot 2012$ S. Karger AG, Basel
The Fox genes are members of the forkhead/winged helix family of transcription factors, which play important roles in a variety of developmental processes, including germ-layer specification, gastrulation, cell-fate determination, and morphogenesis [Carlsson and Mahlapuu, 2002; Pohl and Knöchel, 2005]. Fox genes can be grouped into 18 subclasses named from $\mathrm{A}-\mathrm{Q}$, based on the amino acid sequence of their respective DNA-binding domains, the Forkhead (FH) domain [Kaestner et al., 2000; Shu et al., 2001; Carlsson and Mahlapuu, 2002]. Genomic surveys and embryonic gene expression studies for this gene family have been conducted in both invertebrate and vertebrate organisms [Mazet et al., 2003; Adell and Müller, 2004; Magie et al., 2005; Tu et al., 2006; Yu et al., 2008]. All these studies show that the Fox transcription factors are involved in a variety of biological processes.

Medaka has been used as a model vertebrate for the study of developmental and evolutionary biology. Several of its advantages include a smaller genome (about 800 $\mathrm{Mb}$ ), the existence of polymorphic and highly fertile inbred strains and the fact that they are growth permissive at a wide range of temperatures during embryonic development [Wittbrodt et al., 2002; Naruse et al., 2004]. The

X. Shen and J. Cui contributed equally to this work.

\section{KARGER}

Fax +4161306 1234 E-Mail karger@karger.ch www.karger.com (c) 2012 S. Karger AG, Basel

$1424-8581 / 12 / 1362-0123 \$ 38.00 / 0$

Accessible online at:

www.karger.com/cgr
Jianzhou Cui

Saw Swee Hock School of Public Health

National University of Singapore

16 Medical Drive, Singapore 117597 (Singapore)

Tel. +65 6516 4996, E-Mail jianzhou_cui@nuhs.edu.sg 
availability of a sequenced genome [Kasahara et al., 2007] offers a rich source of information and facilitates genomic studies on the medaka Fox gene family. Until this study, only 5 Fox genes were characterized in medaka [Okamoto et al., 2001; Li et al., 2007]. In the present study, we set out to identify and classify those Fox genes in medaka in order to study aspects of gene evolution and developmental function of the Fox family of genes in this species.

\section{Materials and Methods}

Fish

The inbred strain Hd-rR was used as the experimental model. Developmental stages of the embryos were determined according to criteria described by Iwamatsu [2004].

\section{Identification of Fox Genes}

First we downloaded the sequences of human and zebrafish Fox genes transcription factors from the National Center for Biotechnology Information (NCBI) and ZFIN, respectively. These Fox genes were then used to search for counterpart Fox genes in the medaka genome database by TBLASTX through Ensemble [Hubbard et al., 2005]. The identified sequences were used to search back against the NCBI database by BLASTX to detect matches that were not Fox genes. mRNA from adult tissues and fry (stage 45) were extracted using the RNeasy kit (Qiagen, Germany), and the cDNA was synthesized using the SMART ${ }^{\mathrm{TM}} \mathrm{RACE}$ cDNA Amplification Kit (Clontech) according to manufacturer's instructions. Full or partial length clones were cloned by Rapid Amplification of cDNA Ends (RACE) using the universal primer and the gene-specific primers designed from those sequences we identified from the genome searches. Primer sequences are listed in online supplementary table 1 (for all online suppl. material, see www.karger.com/doi/10.1159/000335898).

\section{Phylogenetic Analysis and Synteny Identification}

For phylogenetic analysis, multiple alignments were performed using ClustalW [Thompson et al., 1997]. The phylogenetic tree was generated with MEGA 5.0 [Tamura et al., 2011] using a maximum likelihood algorithm with a Jones-Taylor-Thornton (JTT) model.

Synteny analyses of FOX genes were performed using information from the genome browsers from human and medaka within the Ensemble database. The Synteny Database [Catchen et al., 2009] was used for large-scale synteny analyses. Generally, 30 $\mathrm{Mb}$ around the human FOX genes were taken into account. For the analysis of paralogous relationships within the medaka genome, human was chosen as the out-group genome.

\section{Spatial and Temporal Expression Patterns}

Quantitative PCR

The mRNA from different medaka tissues including liver, spleen, brain, intestine, eye, ovary, and testes and that of embryos at different stages: $2-8$ cells (stage $3-5$ ), blastula (stage 10), gastrula (stage 15 ), stage 17 , stage 25 , stage 29 , stage 31 , stage 34 , stage 36 , and stage 39 were extracted using the RNeasy kit, and the RNA were reverse-transcribed to cDNA using the Omiscript kit (Qia- gen) according to the manufacturer's protocols after DNase I (Invitrogen) treatment.

Real-time PCR was carried out according to the protocol of Platinum SYBR Green quantitative PCR (qPCR) SuperMix UDG (Invitrogen). $\beta$-Actin was used as an internal control. Data were expressed as the mean \pm SD of 15 replicates. The primer sequences and the product lengths are listed in online supplementary table 1 .

Whole-Mount in situ Hybridization

WISH of medaka embryos was carried out according to protocols described elsewhere [Shinomiya et al., 2000]. Sense and antisense digoxigenin-labeled RNA probes for WISH were synthesized partial length clones of Fox genes with a DIG RNA labeling kit (Roche). The length of probe and the primers used are listed in online supplementary table 1.

\section{Results}

\section{Identification of Fox Transcription Factors in the Medaka Genome}

We found a total of 31 Fox gene sequences by searching the genomic sequence of medaka. Together with the 5 published genes, there are thus 36 Fox genes encoded in the current assembly of the medaka genome. Further, all the newly found Fox genes were cloned using RACE PCR. After performing sequence analyses, these sequences were confirmed and submitted to Genbank (GenBank accession: FJ792649-FJ792679). We further assigned names to these genes from OlFoxA to OlFoxR1 according to the criteria published by the Chordata Fox Nomenclature Committee (http://biology.pomona.edu/ fox/) [Kaestner et al., 2000] and phylogenetic analysis. A summary of medaka Fox genes is shown in online supplementary table 2. Besides the current name, the Genebank Accession number, ORF length and the LG location of the corresponding genes are shown in the table.

\section{Gene Structure and General Features of Fox Genes}

In medaka, the approximate location of the FH domain and the size of the predicted proteins are different for different Fox genes. Among all the complete Fox genes, the smallest protein was FoxL2 (306 aa), the largest, FoxO5, with 668 aa. Besides the FH domain, we also found several other domains in medaka Fox genes by BlastP analysis. For instance, a HNF3_C domain was found at the C-terminal of the proteins in the FoxA subgroup; in Foxk1, the FH-associated domain was found at the N-terminal of the proteins. Most Fox genes have more than one exon; in addition, we found that the position of 
an intron was highly conserved at the N-terminal in the FoxA subclass (data not shown).

\section{Phylogenetic Analysis}

In order to study the phylogenetic relationship of Fox genes in more detail, all of the Fox genes from medaka, human, chicken, frog, zebrafish, and Caenorhabditis elegans were categorized into groups based on phylogenetic analyses of the amino acid sequences. The topology of the resulting neighbor joining tree (online suppl. fig. 1,2) revealed the evolutionary relationships in the Fox gene family. In online supplementary figure 1 , as expected, all the medaka Fox genes can be classified into 18 subclasses, which were named from OlFoxA to OlFoxR1. However, in this study, we classified the OlFoxA3 (gi21263704) and OlFKH1 (NP_001098163) into groups A2 and A3, respectively, which diverged from published results [Okamoto et al., 2001].

A larger phylogenetic tree comparing Fox families from medaka, human, chicken, frog, zebrafish, and $C$. elegans can be found in online supplementary figure 2 . This phylogenetic tree displays the lineage-specific origin and expansion of Fox groups. For example, the FoxG group was represented as a single gene group in C. elegans (CeFox2). Expansion of this group in vertebrates such as chicken, humans and teleosts has resulted in the FoxP group containing 2 genes in medaka.

A summary chart of Fox gene subclasses at different evolutionary stages is shown in online supplementary figure 3. The chart shows the distribution of Fox transcription factors in the 7 species. The top part of the figure shows the phylogenetic relationships among the species. Almost all of the Fox subclasses can be detected among the deuterostomes, except for FoxE, FoxH, FoxR, and FoxS. The FoxI1, FoxJ, FoxL2, FoxM1, and FoxQ1 subclasses can be detected in deuterostomes, but are absent in the ecdysozoans and cnidarian out-groups. Fox subclass FoxH and FoxR could be found only in Chordata, and the FoxS subclass could only be identified in human.

\section{Chromosomal Synteny of Fox Genes}

To examine the synteny of partial Fox genes, we identified the medaka Fox orthologs in human or mouse chromosomes using Synteny Database. The gene traces connecting the fox regions on mouse chromosomes to a region on medaka chromosomes are shown in online supplementary figure 4 . The FoxO gene is a good example to show the full view of gene duplication and evolution in the fish linage, and so we analyzed the synteny mapping of foxO1/3 between medaka and human as follows.

\section{FoxO1/3 Paralogs in the Human Genome}

We examined the origins of FoxO1/3 paralogs in the human genome first. According to the results of BLASTP analysis, FOXO1, located on human chromosome 13 (Hsa13), has a single paralog in the human genome, FOXO3, on chromosome 6 (Hsa6). A paralogy dotplot for Hsa13 (fig. 1A) showed this duplication pattern within a large region encompassing FOXO1. More than $60 \mathrm{Mb}$ of Hsal3 contained genes with paralogs on $\mathrm{Hsa} 6$, spanning the region that includes $F O X O 3$ providing evidence that this region of Hsa13/Hsa6 was produced in a duplication event. Besides the entire genome view in dotplots, a gene trace model also provided a more detailed view of a conserved region (online suppl. fig. 5). The Synteny Database identified a conserved region of 12 pairs of Hsa13/Hsa6 paralogs near FOXO1 using a sliding window size of 50 .

\section{Orthology and Paralogy of Medaka foxo1}

\section{Chromosome Segments}

The ortholog circle plot of figure 1B summarizes the human and medaka syntenic clusters identified by the Synteny Database. So, the gene traces connect the region on Hsa13 with FOXO1 to a region on Ola13 with the foxola gene and the second connecting region on Hsal3 with FOXO1 to a region on Ola14 that does contain foxolb.

An orthology dotplot for Hsa13 clearly showed strong conservation between genes on the long arm of Hsa13 and medaka genes on both chromosomes Ola13 and Ola14 (online suppl. fig. 6A). The Synteny Database identified conserved regions between Hsa13 and both Ola13 and Ola14; a gene-by-gene comparison (fig. 1C) showed 21 pairs of orthologous genes surrounding the FOXO1/ foxola orthologs. Similarly, the orthologous syntenic cluster associated with medaka foxolb has 40 pairs of orthologs between Hsa13 and Ola14 (fig. 1C).

\section{Orthology and Paralogy of Medaka foxo3 \\ Chromosome Segments}

We examined the Synteny Database with an orthology dotplot of Hsa6 which contain FOXO3. The dotplot revealed strong conservation between Hsa6 and Ola11 or Ola16 (online suppl. fig. 6B). Similarly, the gene traces in the circle plot connect the region on $\mathrm{Hsa} 6$ with FOXO3 to a region on Ola11 and Ola16 with foxo3b and foxo $a$ genes, respectively (fig. 1B). The Synteny Database found 2 conserved regions on Hsa6 surrounding FOXO3 and they are orthologous to Ola11 and Ola16 (online suppl. fig. 7). The conserved region between human and medaka synteny block contains 15 and 3 pairs of orthologous genes providing strong support. 

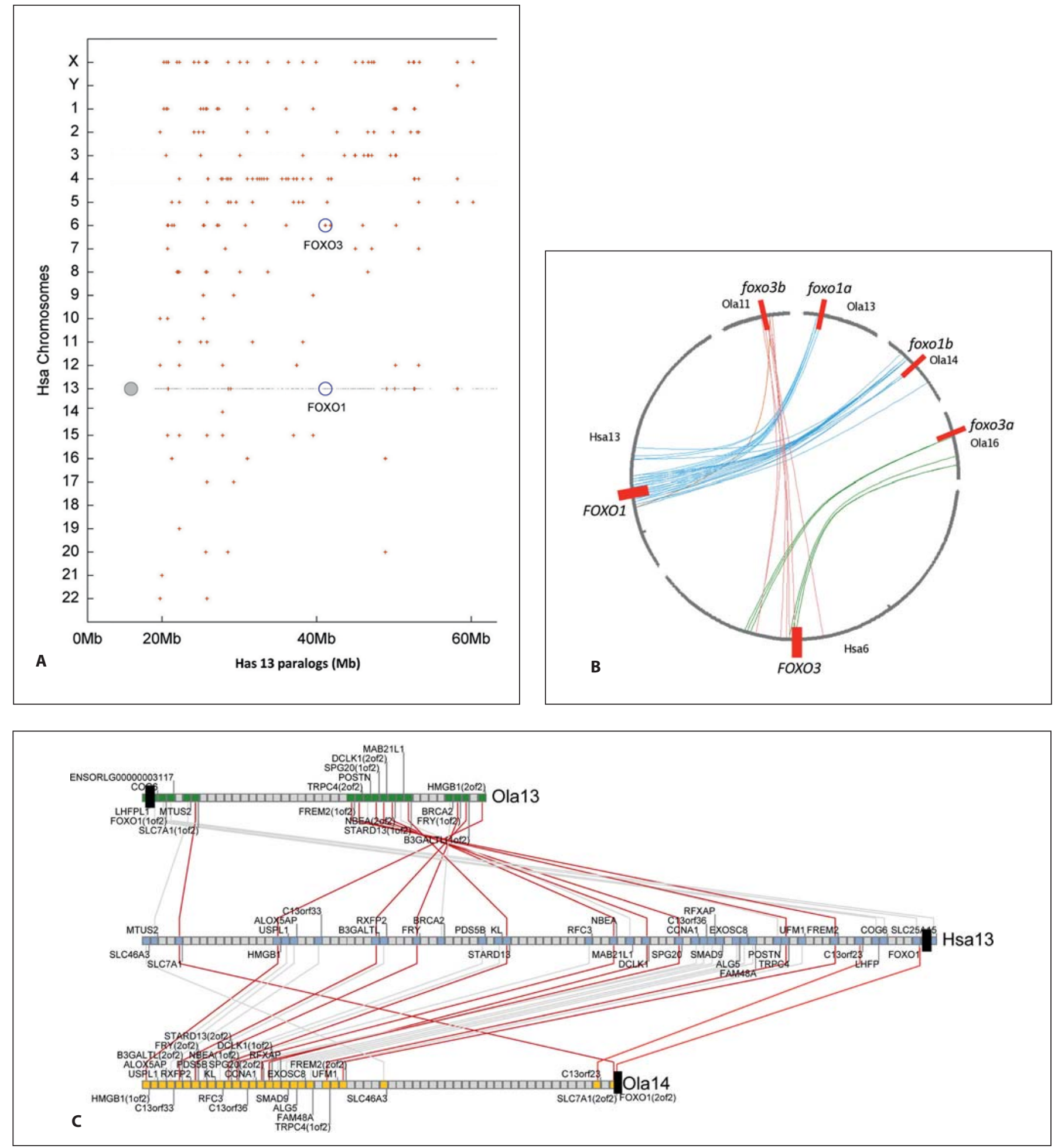

Fig. 1. Conserved syntenies for FOXO1/3 genes. A Human chromosome 13 (Hsa13) paralogy dotplot. Each gene on Hsa13 is represented as a gray dot with its corresponding paralogs. FOXO1 (Hsa13) and FOXO3 (Hsa6) are circled (the centromere is a gray circle). B A circle plot summarizing human and medaka Fox family clusters. Arcs along the circumference of the circle represent chromosomes, while arcs within the circle connect pairs of orthologs. C The FOXO1 orthologous syntenic cluster showing strong syntenic conservation between Hsa13 and Ola13, Hsa13 and Ola14 (50-gene sliding window). The orthologs location was marked by black bars. 


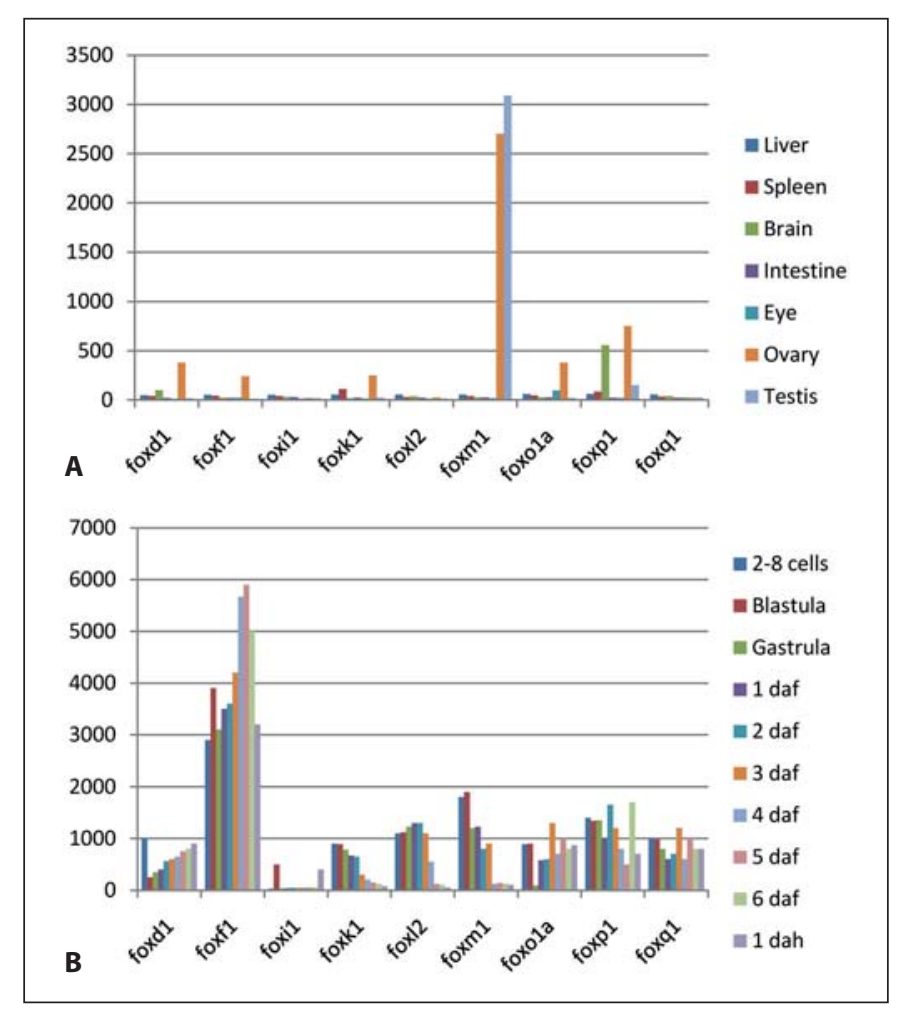

Fig. 2. The expression patterns of partial Fox genes in adult tissues and embryo development stages using qPCR. The relative expression levels (RNA abundance) were calculated by dividing the number of copies of the target gene by that of $\beta$-actin $(\mathbf{A})$, the expression pattern in 7 adult tissues detected by qPCR. B The expression pattern in 10 stages (St. 3-40) of embryo development detected by qPCR. daf = Day after fertilization; dah = day after hatching.

\section{Spatial and Temporal Expression Patterns}

Expression Patterns in Adult Tissues

qPCR results showed that foxd1 was mainly expressed only in the brain and ovary, and the expression level in ovary was 3 -fold higher than that in the brain (fig. 2A). In figure $2 \mathrm{~A}$, the expression of fox $f 1$ could be detected in the ovary and intestine, and the expression level in ovary was 9-fold higher than that in the intestine.

For foxil and foxk1, qPCR results showed that foxil was expressed both in the ovary and intestine, and foxkl mainly in the ovary. For foxil, the relative expression level in the intestine was 15-fold higher than that in the ovary (fig. 2A).

Foxl2 was mainly expressed in the ovary but also in the liver and brain. However, the relative expression level in the liver and brain were 18- to 25-fold lower than that in the ovary (fig. 2A). Foxm1 was mainly expressed in adult ovary and testis, and the expression level of this gene in these 2 tissues is much higher than in other tissues (fig. 2A).

Fox Genes in Medaka
Foxola was expressed in the ovary and eye, and the relative expression level in the ovary was 3 -fold higher than in the eye (fig. 2A). Foxq1 was expressed in the spleen and ovary, and the expression levels between these 2 tissues are similar (fig. 2A).

Unlike other Fox genes we identified, the foxp1 transcription factor was expressed in all the tissues examined in this study, including liver, spleen, intestine, eye, ovary, and testis, with the ovary showing the highest expression level among all the other tissues examined (fig. 2A).

Expression Patterns in Embryonic Development

Similar to the WISH results, qPCR data showed that most of the fox genes are expressed before the gastrula stage, with the exception of foxil. We could not detect foxil at the 8-cell and gastrula stages (fig. 2B, fig. 3G-I). Three genes are expressed at relatively higher levels: these genes are foxfl, foxm 1 (at blastula and gastrula stage) and foxp1 (fig. 2B, fig. 3D-F, P-R, V-X). foxd1 and foxola display low abundance at blastula and 2-8-cell stage, respectively (fig. 2B, fig. 3A-C, S-U).

Seven genes are expressed in the brain: these genes are foxd1, foxf1, foxil, foxk1, foxl2, foxm1, and foxq1. Among the 7 genes, foxd 1 and foxf 1 are also expressed in the gut tube from 1 day after fertilization (daf) to 1 day after hatching (dah) stage (fig. 3C, F), and the relative levels of these 2 transcripts increases steadily until they reached a peak at stage 40, respectively (fig. 2B). Foxd1 transcripts are also detected in the pineal gland at 3 daf (stage 29, fig. $3 \mathrm{~B})$. The remaining 5 genes can be detected by qPCR; however, detection by WISH was not obvious, especially for foxil and foxq1 (fig. 3I, Za).

Foxola was expressed in the olfactory pit and swim (air) bladder, where its expression was detected from 2 daf (stage 25) and 5 daf (stage 34), respectively. The signal intensity of this gene increases steadily in the 2 tissues until 1 dah (stage 40, fig. 3T, U).

Foxp1 was predominantly expressed in the medulla oblongata from 2 daf (stage 25) to 6 daf (stage 39). Transcript number increases until reaching a peak at $4 \mathrm{daf}$ (stage 31 ) before it decreases until 1 dah (stage 40, fig. 3W-X).

\section{Discussion}

\section{Fox Genes in Medaka}

Based on the new nomenclature [Kaestner et al., 2000] and phylogenetic analysis with human FOX genes, the present study revealed the presence of 31 Fox genes in 18 subclasses in the medaka genome (suppl. table 2). We can-

Cytogenet Genome Res 2012;136:123-130 
Fig. 3. The expression patterns of partial Fox genes in 3 embryo development stages range (St. 15, 30 and 40) using WISH. Arrows: positive signal in different locations. $\mathrm{AB}=$ Air bladder; FLM = fasciculus longitudinalis medialis; $\mathrm{GO}=$ gonad; $\mathrm{GT}=$ gut tube; $\mathrm{HC}=$ habenular commissure; $\mathrm{MB}=$ mid-brain; $\mathrm{MO}=$ medulla oblongata; $\mathrm{OP}=$ olfactory pit; $\mathrm{PF}=$ pelvic fin; $\mathrm{PI}=$ pineal gland; $\mathrm{TE}=$ telencephalon.
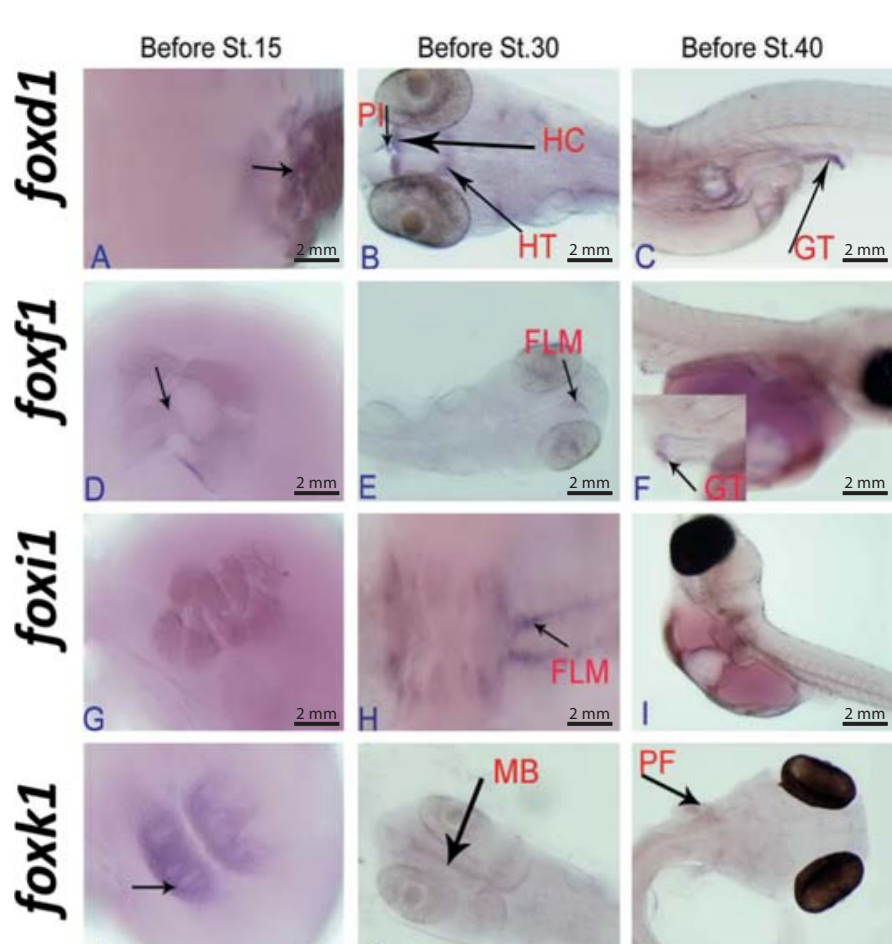

J
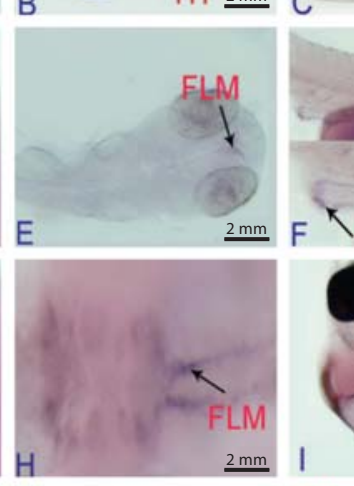

$\underline{2 \mathrm{~mm}}$
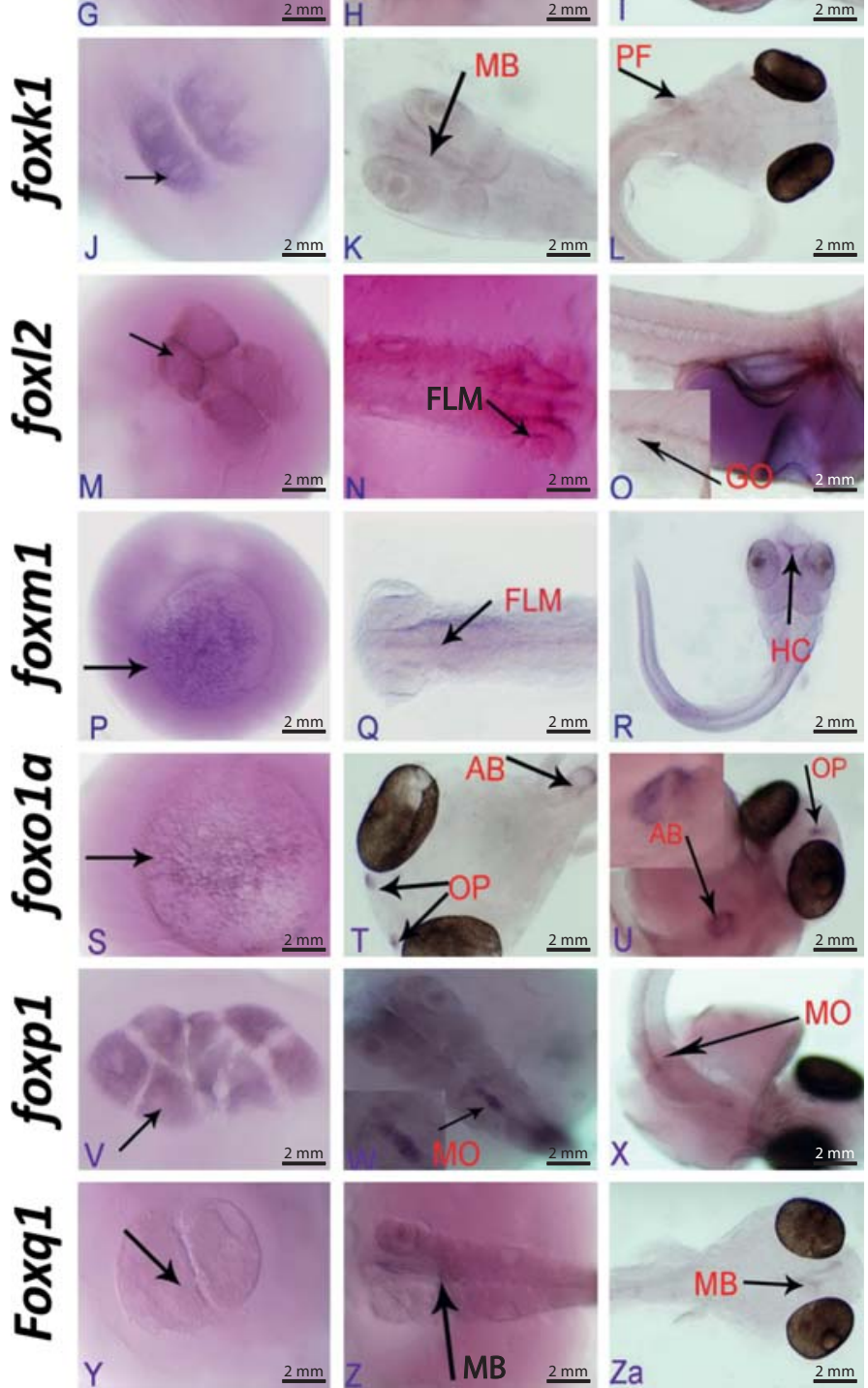
not claim to have identified all the Fox genes in this species, due to possible uneven representation of cDNAs in the mRNA pools, some gaps in whole genome sequences in the database and/or bias originating from the primer sets.

\section{Comparative Genomics of Medaka Fox Loci}

Vertebrate genomes contain numerous duplicate genes, many of which are organized into paralagous regions indicating duplication of linked groups of genes. Retention of linkage between clustered Fox genes observed in humans, amphioxus and some insects has been hypothesized to have been constrained by co-ordinated regulation, analogous to that suggested for the homeobox genes [Mazet et al., 2006]. In medaka, zebrafish and human genomes, we found evidence for Foxq1-Foxf2-Foxc1 linkage; however, only the Foxf1-Foxc2-Foxl1 linkage exists in human, and foxc2 appears to have been lost in the medaka and zebrafish genomes (online suppl. table 3 ). Therefore, our data are consistent with previous results and suggested a break-up of Fox cluster in vertebrates, especially for the teleosts.

\section{The Evolution and Gene Duplication of Fox Genes in} Vertebrates

The hypothesis that teleost fish experienced a third genome duplication after splitting from the lineage that led to humans [Amores et al., 1998; Naruse et al., 2004; Postlethwait et al., 2004] predicts that there should be 2 orthologs (co-orthologs) of each human FOXO1/3 gene in the medaka and other teleosts. To test these predictions, we used the Synteny Database to identify medaka orthologs of human FOXO1 and FOX3 and then used the Synteny Database to search for conserved synteny in regions surrounding those orthologs. The ortholog circle plot of figure $1 \mathrm{~B}$ summarized the human and medaka syntenic clusters identified by the database. Together with the data shown in the figure 1 and other synteny analyses shown in the online supplementary figures, the third genome duplication had been clearly described in terms of FOXO1 and $\mathrm{FOXO} 3$ gene evolution pattern.

\section{Spatial and Temporal Expression Patterns}

FoxD1 was successfully used to convert ectoderm into neural tissue in Xenopus embryos [Mariani and Harland, 1998]. In this study, a high level of foxd1 was detected in the forebrain and pineal gland by WISH from 2-4 daf during medaka development. This expression pattern shows that this gene has a potential role in regulating neural system development. The high expression level in the gut tube indicated that the foxd 1 transcripts are involved in gut development.
During embryonic development, FoxF1 was expressed mostly in the mesenchyme of various organs, such as the digestive, respiratory and urinary tracts [Ormestad et al., 2004]. Similar with foxd1, the expression pattern in the gut shows that this gene has a potential function in gut formation and digestive system development in medaka.

A recent study showed that FoxO1 null embryos exhibit defects in vascular development, indicating that FKHR plays an important role in blood vessel formation [Hosaka et al., 2004]. However, in our study, qPCR results showed that this gene was mainly expressed in the eye and ovary, and WISH data also confirmed that foxola transcripts play an important role in olfactory pit and swim bladder development.

Previous reports showed that FoxP1 mRNA was highly expressed in the striatum projection neurons during the late stage of development [Tamura et al., 2002]. In this study, strong signals can be found in the medulla oblongata from stage 20-40. The expression pattern of foxpl mRNA in medaka suggests that foxp1 may play a role in the development of basal ganglia of the developing and central nervous system.

FOXQ1 has been isolated and characterized in humans where it was strongly expressed in the stomach, trachea, bladder, and salivary gland and weakly expressed in adult and fetal kidney and lung tissue [Bieller et al., 2001]. However, in this study, both qPCR and WISH results only showed that this gene may be involved in spleen and brain developmental processes, indicating that foxq1 expression was compatible with multiple functions of embryonic development.

In summary, we identified the Fox family genes in medaka in the whole genome and investigated expression patterns in different adult tissues and embryonic stages. Synteny analysis results confirmed the lineage-specific evolution in medaka in terms of the Fox gene family. This work will help us to understand the evolution of the whole Fox family and genome duplication in different taxa and the molecular mechanisms for many developmental processes in which these genes are involved.

\section{Acknowledgements}

The authors thank Dr. Tina Sim Huey Fen and Dr. Sebastian Shimeld for critical reading of the manuscript and valuable suggestions. This work was supported by grants from the Japan Society for the Promotion of Science. 


\section{References}

Adell T, Müller WE: Isolation and characterization of five fox (forkhead) genes from the sponge Suberites domuncula. Gene 334:3546 (2004).

-Amores A, Force A, Yan YL, Joly L, Amemiya C, et al: Zebrafish hox clusters and vertebrate genome evolution. Science 282:1711-1714 (1998).

-Bieller A, Pasche B, Frank S, Gläser B, Kunz J, et al: Isolation and characterization of the human forkhead gene FOXQ1. DNA Cell Biol 20:555-561 (2001).

-Carlsson P, Mahlapuu M: Forkhead transcription factors: Key players in development and metabolism. Dev Biol 250:1-23 (2002).

Catchen JM, Conery JS, Postlethwait JH: Automated identification of conserved synteny after whole-genome duplication. Genome Res 19:1497-1505 (2009).

-Hosaka T, Biggs WH 3rd, Tieu D, Boyer AD, Varki NM, et al: Disruption of forkhead transcription factor (foxo) family members in mice reveals their functional diversification. Proc Natl Acad Sci USA 101:2975-2980 (2004).

Hubbard T, Andrews D, Caccamo M, Cameron G, Chen Y, et al: Ensembl 2005. Nucl Acids Res 33:D447-D453 (2005).

- Iwamatsu T: Stages of normal development in the medaka Oryzias latipes. Mech Dev 121: 605-618 (2004).

Kaestner KH, Knöchel W, Martinez DE: Unified nomenclature for the winged helix/forkhead transcription factors. Genes Dev 14:142-146 (2000).

Kasahara M, Naruse K, Sasaki S, Nakatani Y, Qu $\mathrm{W}$, et al: The medaka draft genome and insights into vertebrate genome evolution. Nature 447:714-719 (2007).
-Li J, Iwanami N, Hoa VQ, Furutani-Seiki M, Takahama Y: Noninvasive intravital imaging of thymocyte dynamics in medaka. J Immunol 179:1605-1615 (2007)

Magie CR, Pang K, Martindale MQ: Genomic inventory and expression of Sox and Fox genes in the cnidarian Nematostella vectensis. Dev Genes Evol 215:618-630 (2005).

Mariani FV, Harland RM: XBF-2 is a transcriptional repressor that converts ectoderm into neural tissue. Development 125:5019-5031 (1998).

-Mazet F, Yu JK, Liberles DA, Holland LZ, Shimeld SM: Phylogenetic relationships of the fox (forkhead) gene family in the bilateria. Gene 316:79-89 (2003).

-Mazet F, Amemiya CT, Shimeld SM: An ancient Fox gene cluster in bilaterian animals. Curr Biol 16:R314-R316 (2006).

Naruse K, Hori H, Shimizu N, Kohara Y, Takeda $\mathrm{H}$ : Medaka genomics: a bridge between mutant phenotype and gene function. Mech Dev 121:619-628 (2004).

Okamoto HM, Nakayama I, Nagoya H, Araki K: Predicted protein structure of medaka Foxa3 and its expression in polster. Zool Sci 18: 823-832 (2001).

-Ormestad M, Astorga J, Carlsson P: Differences in the embryonic expression patterns of mouse Foxf1 and -2 match their distinct mutant phenotypes. Dev Dyn 229:328-333 (2004).

Pohl BS, Knöchel W: Of fox and frogs: Fox (fork head/winged helix) transcription factors in Xenopus development. Gene 344:21-32 (2005).

Postlethwait J, Amores A, Cresko W, Singer A, Yan YL: Subfunction partitioning, the teleost radiation and the annotation of the human genome. Trends Genet 20:481-490 (2004).
Shinomiya A, Tanaka M, Kobayashi T, Nagahama Y, Hamaguchi S: The vasa-like gene, olvas, identifies the migration path of primordial germ cells during embryonic body formation stage in the medaka, Oryzias latipes. Dev Growth Differ 42:317-326 (2000).

Shu DG, Chen L, Han J, Zhang XL: An Early Cambrian tunicate from China. Nature 411: 472-473 (2001).

Tamura K, Peterson D, Peterson N, Stecher G, Nei M, Kumar S: MEGA5: molecular evolutionary genetics analysis using maximum likelihood, evolutionary distance, and maximum parsimony methods. Mol Biol Evol 28: 2731-2739 (2011).

-Tamura S, Morikawa Y, Miyajiima A, Senba E: Expression of oncostatin $\mathrm{M}$ in hematopoietic organs. Dev Dyn 225:327-331 (2002).

-Thompson JD, Gibson TJ, Plewniak F, Jeanmougin F, Higgins DG: The CLUSTAL_x windows interface: flexible strategies for multiple sequence alignment aided by quality analysis tools. Nucl Acids Res 25:4876-4882 (1997).

Tu Q, Brown CT, Davidson EH, Oliveri P: Sea urchin forkhead gene family: phylogeny and embryonic expression. Dev Biol 300:49-62 (2006).

Weigel D, Seifert E, Reuter D, Jäckle H: Regulatory elements controlling expression of the Drosophila homeotic gene fork head. Embo J 9:1199-1207 (1990).

Wittbrodt J, Shima A, Schartl M: Medaka - a model organism from the far east. Nat Rev Genet 3:53-64 (2002).

-Yu JK, Mazet F, Chen YT, Huang SW, Jung KC, Shimeld S: The Fox genes of Branchiostoma floridae. Dev Genes Evol 218:629-638 (2008). 